\title{
CCD observations of Triton ${ }^{\star}$
}

\author{
C.H. Veiga and R. Vieira Martins \\ Observatório Nacional, Rua Gal. José Cristino 77, 20921-400 Rio de Janeiro, Brazil \\ e-mail: cave@on.br or rvm@on.br
}

Received January 4; accepted April 11, 1996

\begin{abstract}
Astrometric positions of the Neptunian Satellite Triton are given for the opposition of Neptune for the years 1989 to 1994 . These positions where measured on 433 CCD frames obtained at the Cassegrain focus of a $1.6 \mathrm{~m}$ reflector. They are compared with theoretical positions calculated from Jacobson et al. (1991). The observed minus calculated residuals referred to Neptune have standard deviations of the order of $0{ }^{\prime \prime} 11$.
\end{abstract}

Key words: planets and satellites — satellites of Neptune — astrometry

\section{Introduction}

In the continuation of our systematic program of astrometric observations of natural satellites initiated in 1982, we report in this paper observations carried out during 29 nights in the period from 1989 to 1994 when we obtained 433 CCD frames of the Neptunian satellite Triton. This program was motivated by the present southern declination of the planet which permits observations close to the zenith from our latitude $\left(\phi \approx-23^{0}\right)$. Our previous observations, made from 1982 to 1988, used photographic plates. These observations of Triton were published in a previous paper (Veiga et al. 1995) which present 53 positions of the satellite distributed over 13 nights.

This paper is organized as follows: in Sect. 2 we describe the observations and reduction procedures; in Sect. 3 we compare our results with theoretical calculated positions. A conclusion is presented in Sect. 4.

\section{The observations and measures}

The observations were made at the Cassegrain focus of the $1.6 \mathrm{~m}$ reflector of the Laboratório Nacional de Astrofísica, Brazil, where the scale at the focal plane is $133^{\prime \prime} 0 / \mathrm{mm}$, and we refer the reader to Veiga et al. (1987) for more details about the telescope. From 1988 to July 1992 we used the EEV P86231 CCD which is a array with $385 \times 578$ squares pixel each pixel measuring $22 \mu \mathrm{m}$ which corresponds to 0 '! 288 on the sky. This chip was replaced by an EEV P88231 of $770 \times 1152$ square pixel with 22.5 $\mu \mathrm{m}$. No filter was used and the exposure time varied from

Send offprint requests to: C.H. Veiga

*Based on observations made at Laboratório Nacional de Astrofísica/CNPq/MCT-Itajubá-Brazil
3 to 10 seconds, depending on the meteorological conditions. The distribution of our observations during the six years are shown in Fig. 1.

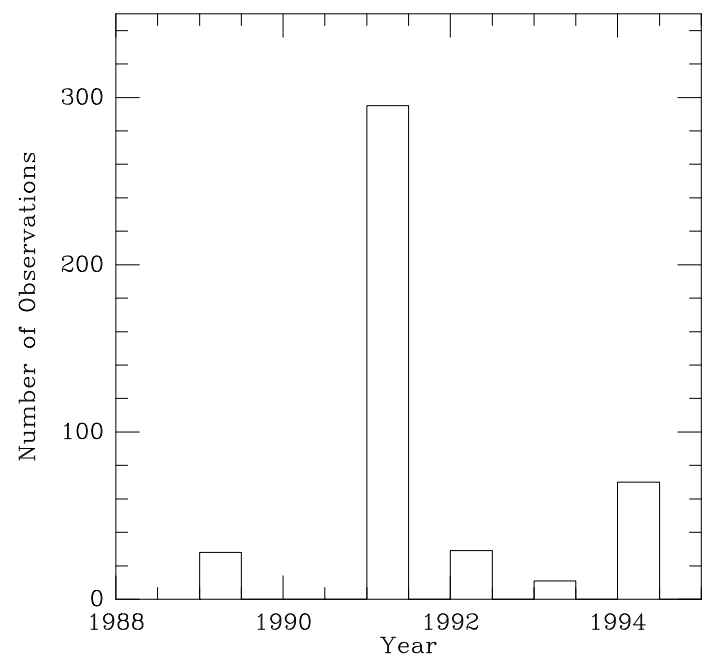

Fig. 1. The distribution of the observations

To find the centers of star and satellite images we used the program ASTROL (Colas \& Serrau 1993) which fits simultaneously a bi-dimensional Gaussian to every image and that considers a second degree polynomial for the sky background. For a discussion on the determination of astrometric centers of satellite images, see Veiga \& Vieira Martins (1995a). The error in the centering for Triton is 0. "02 and for Neptune $00^{\prime \prime} 04$ (Neptune has magnitude 7.7 and Triton 13.5).

The astrometric calibration of almost all frames was obtained through the method described by Veiga \& Vieira 
Martins (1994) using the motion of Neptune. For 30 frames where the method of the motion of the planet could not be used, the scale and the direction were obtained by the frames of Uranian satellites observed in the same night. For these images of the Uranian system, the scale and the direction of the frame were determined using the satellites Ariel, Umbriel, Titania and Oberon and the theoretical positions given by GUST86 (Laskar \& Jacobson 1987). These observations of the Uranian satellites have good accuracy (Veiga \& Vieira Martins 1995b). To compare both methods, for all frames where they could be applied, the scales and the directions were compared and the relative errors in the positions of Triton were less than $10 \%$ of the error given by one of the methods.

Our residuals in $X\left((\mathrm{O}-\mathrm{C})_{x}\right.$ for Triton minus Neptune positions) present a systematic error which increases with the zenithal distance $(z)$. Their mean values decrease from $0^{\prime \prime} 02$ to $-0^{\prime \prime} 06$ when $z$ goes from $0^{0}$ to $50^{0}$. Otherwise the $(\mathrm{O}-\mathrm{C})_{y}$ decreases from $0 . \prime 05$ to $0{ }^{\prime \prime} 01$. Probably, this error is due to the different color between Neptune \& Triton (Wamsteker 1973; Cruikshank et al. 1979; Jacobson 1990) combined with the CCD sensitivity with wavelength and the atmospheric condition during our observations. Then, we computed the total astronomical refraction separately for Neptune and Triton considering a difference of 0.'19 between their constant of refraction and after by the difference of the corrected positions we obtained the new positions of satellite related to the planet. Considering the typical conditions for pressure and temperature during our observations this $00^{\prime \prime} 19$ (which is $00^{\prime \prime} 15$ after the corrections corresponding to the pression and the temperature during our observations) corresponds to a difference of 0. . 07 $\mu \mathrm{m}$ between the effective wavelength observed for Neptune and Triton (Allen 1973) which fits well our estimated values: $0.60 \mu \mathrm{m}$ for Neptune and $0.67 \mu \mathrm{m}$ for Triton. After these corrections, the mean residuals in $X$ decrease from $0^{\prime \prime} 02$ to $-0^{\prime \prime} .01$ when $z$ goes from $0^{0}$ to $50^{0}$. This correction is important because there are $24 \%$ of the observations with zenithal distances greater than $30^{\circ}$.

In Table 1 (accessible in electronic form) we list the observed positions of Triton relative to Neptune. The data are presented in the following form: the first line gives the year, month and day and decimal fractions of UTC days, corresponding to the mean instant of the observation. In the next line we list the name of the satellite followed by $X(\Delta \alpha \cos \delta)$ and $Y(\Delta \delta)$ in arcseconds of Triton, referred to Neptune. The reference system is defined by the mean equator and equinox 1950.0. Only the positions with residuals better than $3 \sigma$ after a first reduction where selected.

\section{Comparison between observations theoretical positions}

We compared the measured with theoretical positions of Triton which are calculated using the parameters given by Jacobson et al. (1991) (Table 5). The mean residuals $(\mathrm{O}-\mathrm{C})$ are $\bar{x}=-0^{\prime \prime} \cdot 001$ and $\bar{y}=0^{\prime \prime} \cdot 032$ and the standard deviations are $\sigma_{x}=00^{\prime \prime} 093$ and $\sigma_{y}=00^{\prime \prime} 116$. The errors, two times greater than those in the positions of Uranian satellites (Veiga \& Vieira Martins 1995b) are probably caused by the error in the center of Neptune's image as remarked above.

In Fig. 2, we present the residuals of Triton as function of the date of observation. Our residuals from photographic data were also plotted (Veiga et al. 1995). We can see that these residuals are uniform for the 10 years showing a good agreement with the Jacobson ephemeris. The residuals of CCD observations as a function of the longitude of the satellite are shown in Fig. 3.
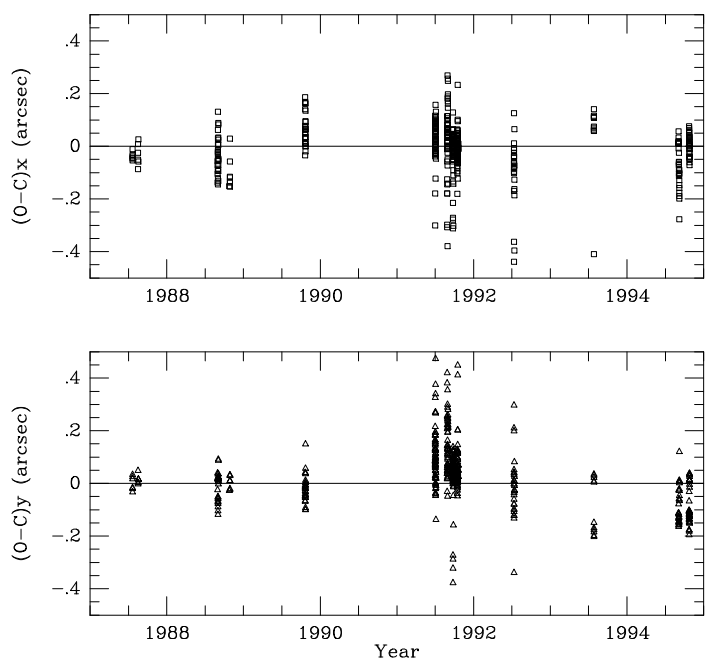

Fig. 2. The (O-C) of Triton as function of the time
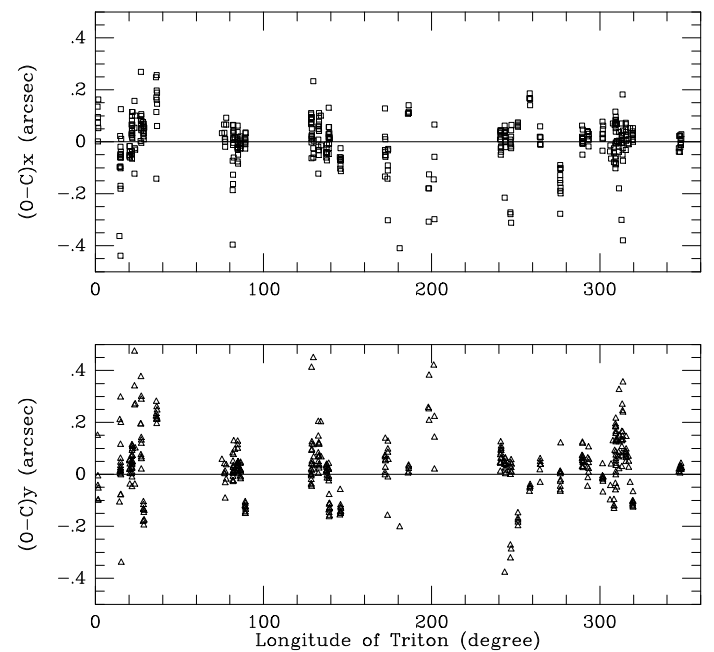

Fig. 3. Residuals for Triton referred to Neptune versus the longitude 


\section{Conclusion}

We presented above 433 CCD observations of Triton made with the same telescope and reduced by the same methods. They are distributed between 29 nights in 6 years and their errors is about $00^{\prime \prime} 11$. If we consider that, from more than 2000 observed positions of Triton published after its discovery by Lassel in 1846, only about 300 have an accuracy better than 0"15 (Jacobson et al. 1991), we can conclude that this is an important set of observations of this satellite.

\section{References}

Allen C.W., 1973, Astrophysical Quantities. University of London. The Athlone Press. Third Edition
Colas F., Serrau M., 1993, Astrol et Interpol version 3.10 Edition du Bureau des Longitudes Paris - France

Cruikshank D.P., Stockton A., Dyck H.M., Becklin E.E., Macy Jr. W., 1979, Icarus 40, 104

Jacobson R.A., 1990, A\&A 231, 241

Jacobson R.A., Reidel J.E., Taylor A.H., 1991, A\&A 247, 565

Laskar J., Jacobson R.A., 1987, A\&A 188, 212

Veiga C.H., Vieira Martins R., Veillet C., Lazzaro D., 1987, A\&AS 107, 551

Veiga C.H., Vieira Martins R., 1994, A\&AS 107, 551

Veiga C.H., Vieira Martins R., 1995a, A\&AS 111, 387

Veiga C.H., Vieira Martins R., 1995b, A\&AS 113, 557

Veiga C.H., Vieira Martins R., Le Guyader Cl., Assafin M., 1995, A\&AS 115, 319

Wamsteker W., 1973, AJ 95, 1007 